\title{
Miniaturized Switchless Pattern Reconfigurable Antenna Utilizing Spherical Modes Analysis
}

\author{
Adam Narbudowicz \\ Technological University Dublin, adam.narbudowicz@mydit.ie \\ Max Ammann \\ Technological University Dublin, max.ammann@tudublin.ie \\ Nicola Marchetti \\ CONNECT Centre for Future Networks and Communications, Trinity College, Dublin
}

Follow this and additional works at: https://arrow.tudublin.ie/ahfrccon

Part of the Electrical and Electronics Commons

\section{Recommended Citation \\ Narbudowicz, A., Ammann, M.J. \& Marchetti, N. (2018). Miniaturized Switchless Pattern Reconfigurable Antenna Utilizing Spherical Modes Analysis. 12th European Conference on Antennas and Propagation, EuCAP 2018, London, 9-13th April 2018. doi:10.1049/cp.2018.0568}

This Conference Paper is brought to you for free and open access by the Antenna \& High Frequency Research Centre at ARROW@TU Dublin. It has been accepted for inclusion in Conference Papers by an authorized administrator of ARROW@TU Dublin. For more information, please contact arrow.admin@tudublin.ie, aisling.coyne@tudublin.ie,gerard.connolly@tudublin.ie.

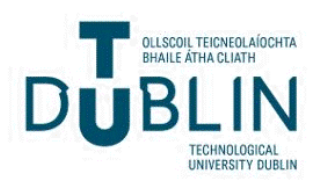




\title{
Miniaturized Switchless Pattern Reconfigurable Antenna Utilizing Spherical Modes Analysis
}

\author{
Adam Narbudowicz ${ }^{1}$, M. J. Ammann ${ }^{2}$ and Nicola Marchetti ${ }^{3}$ \\ ${ }^{1}$ Antenna \& High Frequency Research Centre, Dublin Institute of Technology, Dublin, Ireland, adam.narbudowicz@dit.ie \\ ${ }^{2}$ Antenna \& High Frequency Research Centre, Dublin Institute of Technology, Dublin, Ireland, max.ammann@dit.ie \\ ${ }^{3}$ CONNECT Centre, Trinity College Dublin, the University of Dublin, Dublin, Ireland, nicola.marchetti@tcd.ie
}

\begin{abstract}
The paper proposes an electrically small pattern reconfigurable antenna, which allows flexible beamforming with the antenna diameter being $\lambda / 5$. The antenna has 3 ports, each designed to radiate a different spherical mode. By applying appropriate phase shift the antenna allows to flexibly sweep its bi-directional radiation pattern across a $360^{\circ}$ azimuth, while offering $5 \mathrm{dBi}$ directivity.
\end{abstract}

Index Terms - antenna, electrically small antenna, antenna miniaturization, beamsteering, spherical modes.

\section{INTRODUCTION}

The ever growing need for miniaturized electronics creates a great demand for small antennas, especially for the incorporation into the Internet of Things systems. The basic principles of electrically small antennas with simple, omnidirectional radiation patterns have been well studied in the literature. There are many designs available and the limitations are well known [1 - 4]. However there is still limited understanding on efficient design of electrically small pattern reconfigurable antennas.

Typical solutions for pattern reconfiguration involve the use of switching component (MEMS switches, pin-diode, etc.) to reroute the current in the aperture [5, 6]. Such techniques lack flexibility and support only limited degree of miniaturization. On the other hand antenna arrays allow a very high degree of control over the radiation pattern, but the requirement for spacing between elements practically prevents any miniaturization.

This paper investigates a solution to this problem. It proposes an antenna where the phase shift required for beamsteering is generated by the intrinsic radiation mechanism, rather than by the spacing between antenna elements. This approach allows substantial miniaturization: the proposed antenna has diameter of $\lambda / 5$ and offers similar beamsteering capabilities as a 3-element array with up to $5 \mathrm{dBi}$ directivity. It is based on the principle described in $[7,8]$, however offers substantial miniaturisation and improved beamsteering as compared to [8].

\section{SPHERICAL MODES}

Spherical modes offer a convenient technique to fully describe and analyze the radiation pattern of any antenna. Any radiation pattern of any antenna can be accurately

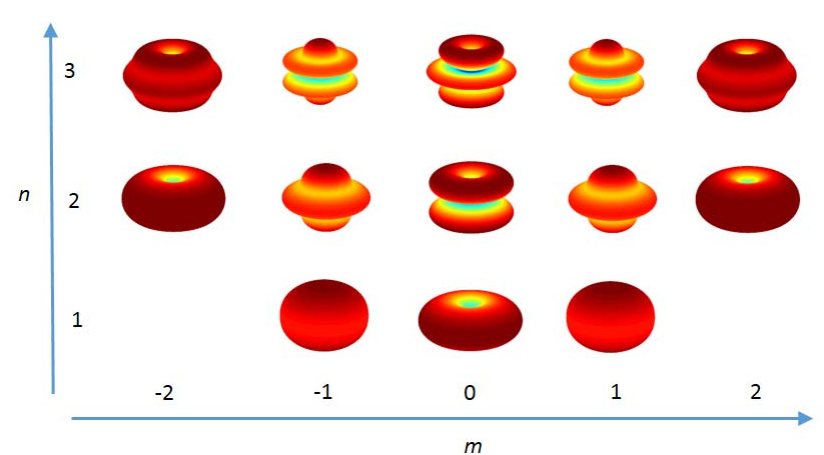

a)

$(1,-2,2) \quad(1,0,1)$

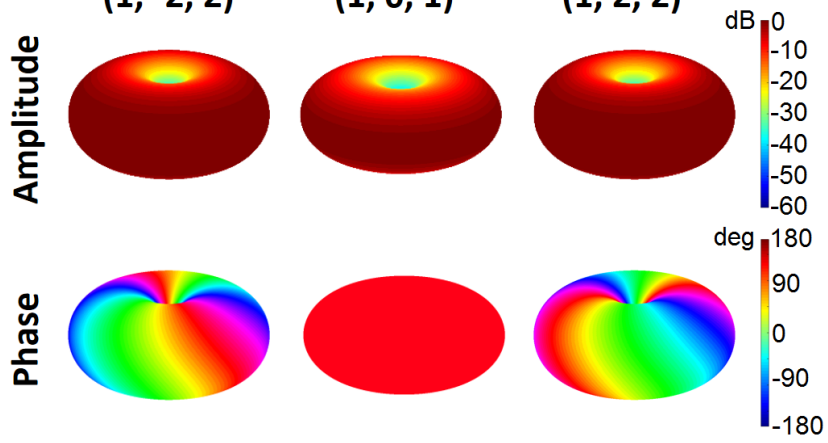

b)

Fig. 1. Visualization of the electric field of spherical modes: a) amplitudes of 13 lowest modes when $s=1 ; \mathrm{b})$ amplitudes and phases of modes $(1,-2$, $2),(1,0,1)$ and $(1,2,2)$ respectively left to right.

represented in the near-field by electric and magnetic fields on a sphere that fully encloses the investigated antenna. Any such distribution of electric and magnetic fields can be separated into a superposition of discreet spherical modes that occur on the sphere surface. Although in general there is a countably infinite number of such modes, in practical terms the number of modes is limited by the size of the sphere compared to the wavelength [9].

In this paper we will denote the modes as $(s, m, n)$, where $s$ is related to the polarization and $m$ denotes the phase variation around the horizontal cut of the modes, i.e. how many times it changes from 0 to $2 \pi$. Within this nomenclature mode $(1,0,1)$ which can be associated with the ideal dipole antenna, while mode $(2,0,1)$ - with the ideal loop antenna. Fig. 1a shows the electric field amplitude of the 13 lowest modes when $s=1$. The three modes of interest 
for this paper are $(1,-2,2),(1,0,1)$ and $(1,2,2)$ depicted in more detail in Fig. 1b. All three modes exhibit very similar amplitudes, corresponding to omnidirectional radiation patterns. However the phase variation in each mode progresses linearly, with the phase of the $(1,0,1)$ mode being constant over the whole sphere, while modes $(1,-2,2)$ and $(1,2,2)$ observe the phase around their circumference to change twice from 0 to $2 \pi$ in a clockwise or counterclockwise direction, subject to the sign of index $m$. This property is crucial for combining flexible array-like beamsteering with size miniaturization. A superposition of those modes with different phase shifts will cause constructive and destructive interferences for different angles. Therefore the principle can be used to direct the beam of the antenna into desired direction, in a similar manner as with classical array. The main challenge of this work is therefore to design a practical and compact antenna structure that will allow realization of those three modes.

\section{DESIGN}

\section{A. Antenna}

The antenna is a cylindrical structure, divided into three parts as seen in Fig. 2. The top part (section A-A' in Fig 2) is a copper monopole of height $H_{m}=10.7 \mathrm{~mm}$ and diameter $D_{m}=10 \mathrm{~mm}$. To provide good matching it is lifted

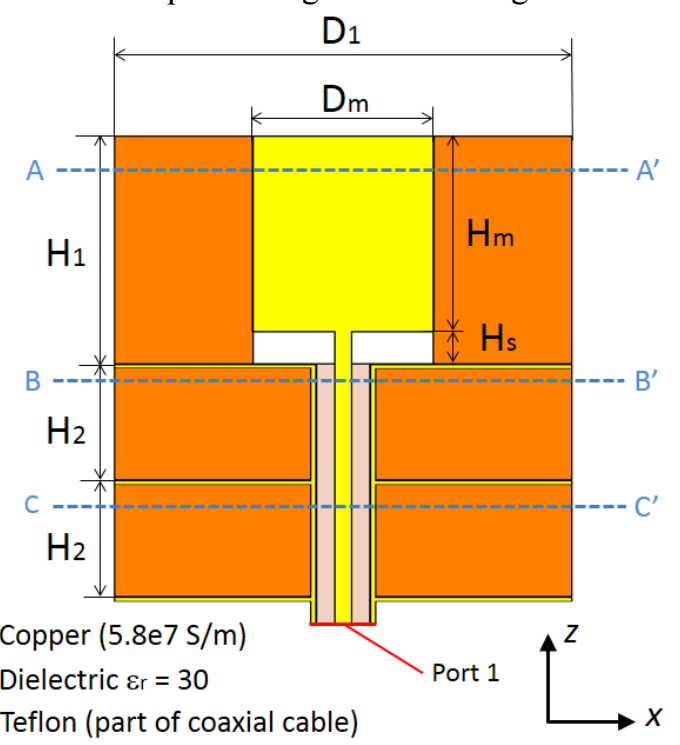

a)

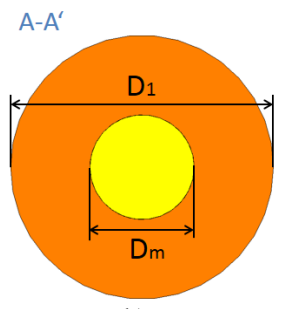

b)

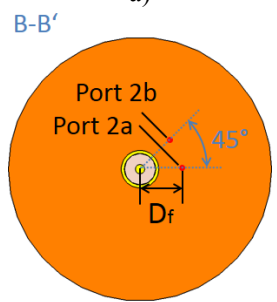

c)

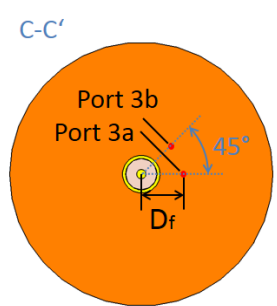

d)
Fig. 2. Proposed antenna: a) cut in $x z$-plane (vertical) with key parameters; b) horizontal cut along A-A' line (top section); c) horizontal cut along BB' line (middle section); d) horizontal cut along C-C' line (bottom section).
$H_{S}=1.8 \mathrm{~mm}$ above the circular copper ground plane of diameter $D_{l}=25.2 \mathrm{~mm}$. The monopole is fed via a coaxial feed line of $50 \Omega$. For miniaturization the monopole is surrounded by a dielectric cylinder of diameter $D_{1}=25.2$ $\mathrm{mm}$ and relative permittivity $\varepsilon_{\mathrm{r}}=30$.

The middle and lower parts (sections B-B' and C-C') are the same, with the sole difference coming from the feed network described in the subsequent subchapter. They are dielectric discs of height $\mathrm{H}_{2}=6.35 \mathrm{~mm}$ supporting two orthogonal $\mathrm{TM}_{21}$ resonant modes. Each of the two modes in each of the parts is fed through a discreet port located $D_{f}=4 \mathrm{~mm}$ from the center of the disc. The two ports within each patch are oriented at $45^{\circ}$ and labeled as ports $2 a$ and $2 b$ for the middle part (cut B-B' seen in Fig. 2c) and port $3 a$ and port $3 b$ for the lowest part (cut C-C' seen in Fig. $2 \mathrm{~d}$ ). The center section of both discs is isolated from the dielectric discs in order to support the coaxial line to feed the top part. This has little impact on the performance of lower parts, as the $\mathrm{TM}_{21}$ mode exhibits a null in its center.

The overall antenna dimensions are: $D_{l}=25.2 \mathrm{~mm}$; $D_{m}=10 \mathrm{~mm} ; H_{l}=12.5 \mathrm{~mm} ; H_{2}=6.35 \mathrm{~mm} ; H_{m}=10.7 \mathrm{~mm}$ and $H_{S}=1.8 \mathrm{~mm}$.

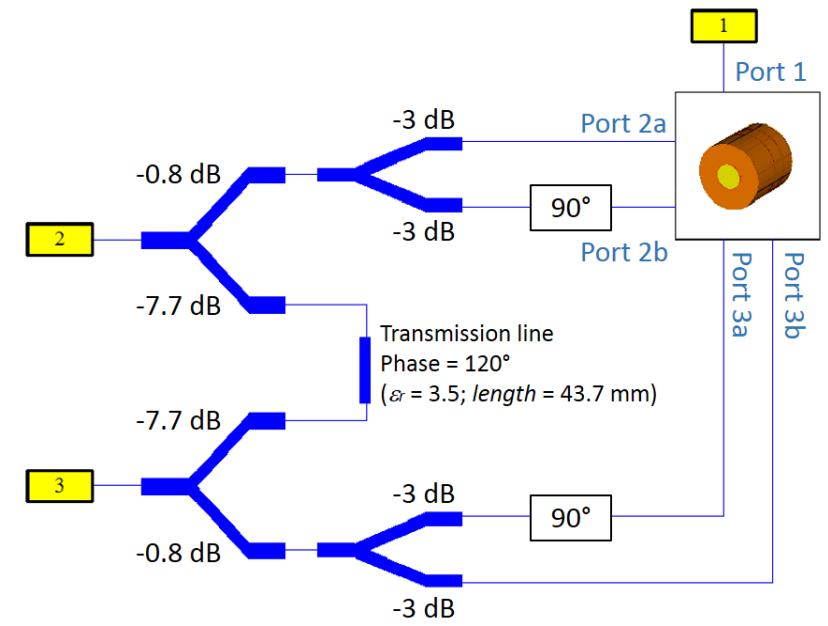

Fig. 3. Feed circuits for the proposed antenna.

\section{B. Feed network}

Fig 3 shows the feed network of the proposed antenna. Port 1 of the feed network is connected directly to the port 1 of the antenna, i.e. the coaxial line protruding from the antenna's lower part.

In order to radiate spherical modes $(1,-2,2)$ and $(1,2,2)$ the two ports need to be fed with equal amplitudes and $90^{\circ}$ phase shift. This is executed by $3 \mathrm{~dB}$ power dividers and phase shifters. For the middle part of the antenna, the phase shifter is connected to the port $2 b$, while for the lower part to the port $3 \mathrm{a}$. This is to ensure the phase in those parts is rotating in opposing directions.

Due to the limited volume of the antenna a significant coupling between the middle and lower parts was detected. Such a coupling is a significant problem, as it destroys the purity of the radiated spherical modes: the modes are by definition orthogonal to each other, therefore in the ideal 


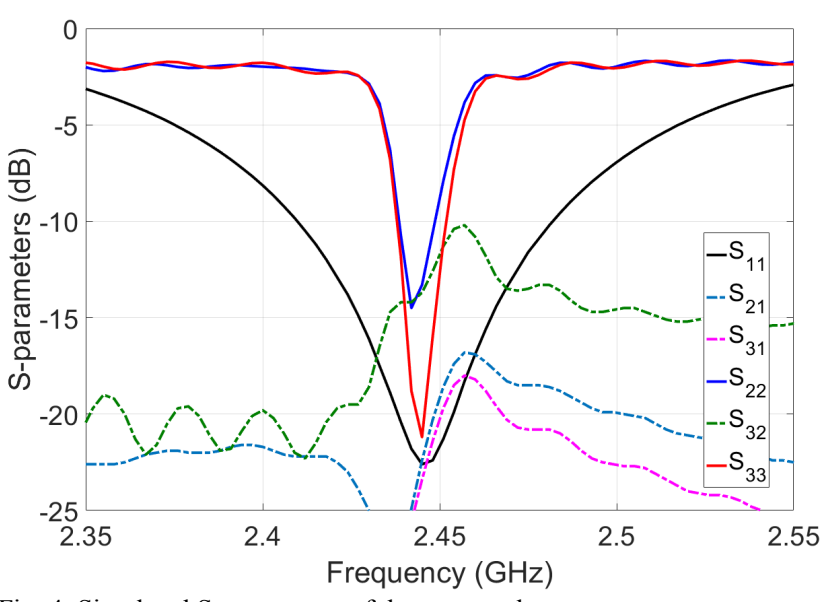

Fig. 4. Simulated S-parameters of the proposed antenna.

case there should be no coupling. If coupling exists it indicates the presence of additional undesired spherical modes that are being excited by the antenna. To combat this problem a coupling cancelation line (seen in Fig. 3 connecting ports 2 and 3 with $120^{\circ}$ phase shift) was added to the circuit. It draws $-7.7 \mathrm{~dB}$ from the initial signal, which is proportional to the coupling at $2.445 \mathrm{GHz}$, and delivers it to

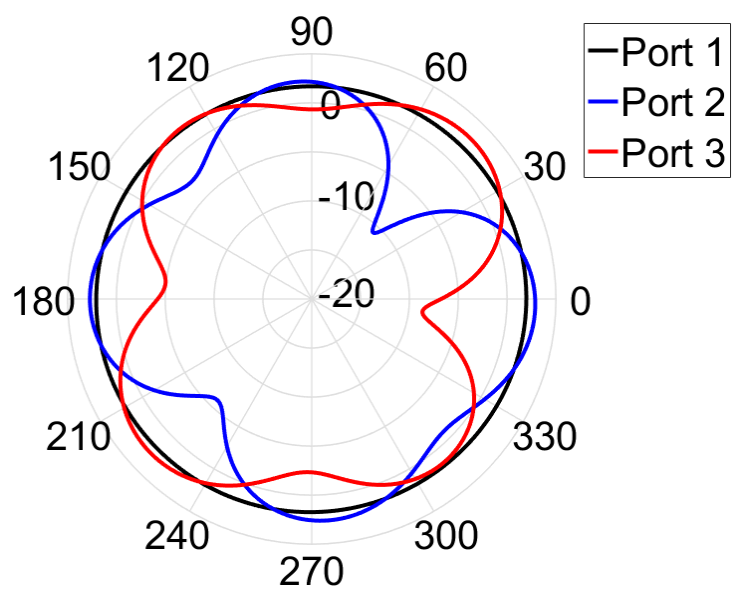

a)

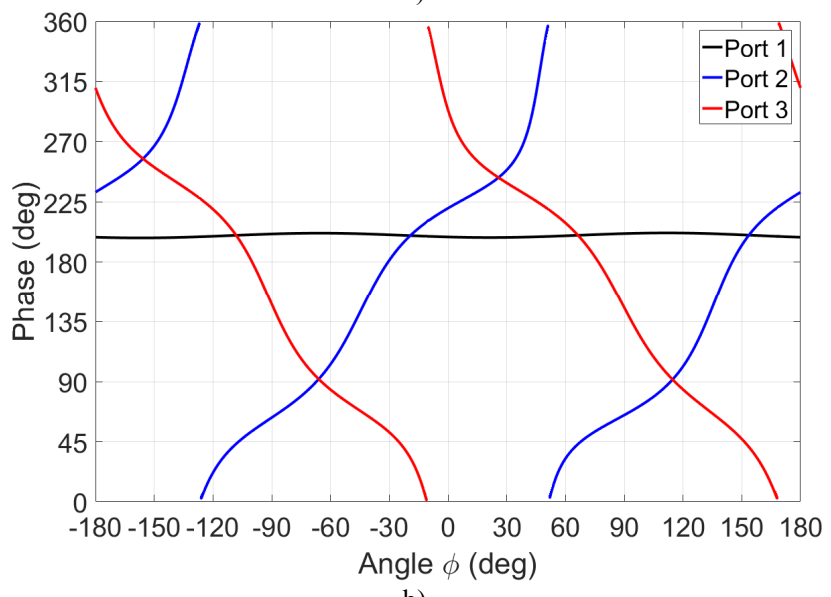

b)

Fig. 5. Directivities of the proposed antenna at $2.445 \mathrm{GHz}$ (vertical polarization): a) amplitudes; b) phases in the far-field. the other port with phase shift equivalent to the coupling plus $180^{\circ}$. Since the antenna is narrowband this simple mechanism is sufficient to achieve good decoupling.

\section{RESULTS}

Fig. 4 shows the S-parameters of the proposed antenna measured at the ports of the feed network. The center frequency is $2.445 \mathrm{GHz}$. The top part radiating $(1,0,1)$ mode exhibits the highest $-10 \mathrm{~dB}$ bandwidth of $72 \mathrm{MHz}$, which is expected. The two parts executing higher order spherical modes achieve $-10 \mathrm{~dB}$ bandwidths of $10 \mathrm{MHz}$ and $14 \mathrm{MHz}$ for ports 2 and 3 respectively. These narrow bandwidths are expected due to the size reduction of the antenna and the use of higher order spherical modes which require more space. Due to the presence of the decoupling line the isolation between ports 2 and 3 is better than $12 \mathrm{~dB}$ within the whole impedance matched bandwidth.

For port 1 the simulated radiation efficiency is $88 \%$, while it drops to $25 \%$ for ports 2 and 3 . This is again expected, as the sphere supporting higher modes needs to be greater than the one supporting the basic mode $(1,0,1)$. Therefore problems known to occur for electrically small antennas - such as increased losses and limited bandwidths will be visible for antennas of greater dimensions if they radiate higher spherical modes.

Fig. 5 shows the radiation patterns when exciting each individual antenna port. It can be seen that while the patterns for ports 2 and 3 are orthogonal to each other, there exist some shouldering and dipping in the amplitude, corresponding to the presence of undesired modes. However the desired phase characteristics have been achieved, with the top part exhibiting constant phase while the two other antenna parts exhibit approximately linear phases changing in opposite directions with angle $\phi$. As will be demonstrated in the subsequent section, this is the key enabler for radiation pattern control with a small antenna.

\section{PATTERN SYNTHESIS}

In contrast to traditional linear antenna arrays for the proposed antenna the relationship between phase and beam direction is linear [10]. Also in contrast to an array the two beams generated are symmetrical with respect to the point, not to the plane as for linear array.

To test antenna performance four different bi-directional beams were generated with main beams separated by $45^{\circ}$. The phase shifts used were dictated by the phase performance shown in Fig. 5b and are shown in Table I. Due to the highest efficiency of the monopole, port 1 was excited with only 0.36 of the power of the other ports. It can be seen in Fig. 6 that despite the imperfection in individual patterns, a very good control and linear beam-steering can be achieved. The main beams have $5 \mathrm{dBi}$ directivity with $66^{\circ}-$ $69^{\circ}$ half-power beamwidth. 
TABLE I.

\begin{tabular}{|c|c|c|c|}
\hline \multirow{2}{*}{ Beam direction } & \multicolumn{3}{|c|}{ Phase shift applied } \\
\cline { 2 - 4 } & Port 1 & Port 2 & Port 3 \\
\hline $0^{\circ} / 180^{\circ}$ & $0^{\circ}$ & $-34^{\circ}$ & $-112^{\circ}$ \\
\hline $45^{\circ} / 225^{\circ}$ & $0^{\circ}$ & $-107^{\circ}$ & $-27^{\circ}$ \\
\hline $90^{\circ} / 270^{\circ}$ & $0^{\circ}$ & $136^{\circ}$ & $56^{\circ}$ \\
\hline $135^{\circ} / 315^{\circ}$ & $0^{\circ}$ & $59^{\circ}$ & $133^{\circ}$ \\
\hline
\end{tabular}

\section{CONCLUSIONS}

The paper demonstrates an electrically small antenna that allows flexible pattern reconfiguration similar to a small array. The antenna diameter is $\lambda / 5$ and despites its small size, it offers a directivity of $5 \mathrm{dBi}$ and the capability to flexibly sweep the beam in the horizontal plane. The price to be paid is the narrow band and increased losses, which is typical for electrically small antennas. For traditional electrically small antennas, which use the lowest modes $(1,0,1)$ and/or $(2,0,1)$, those problems would occur for much higher degree of miniaturization. However the higher modes require a sphere of much greater diameter in order to accommodate the phase variation around its perimeter.

\section{ACKNOWLEDGMENT}

The authors are grateful to Rasmus Cornelius of RWTH Aachen University in Germany who kindly provided the software for the visualization of spherical modes.

This project has received funding from the European Union's Horizon 2020 research and innovation programme under the Marie Skłodowska-Curie grant agreement No 713567 and from Science Foundation Ireland (SFI) under Grant Number 13/RC/2077.

\section{REFERENCES}

[1] S. Best, "The radiation properties of electrically small folded spherical helix antennas", IEEE Transactions on Antennas and Propagation, vol. 52, issue 4, pp. 953 - 960, Apr. 2004.

[2] M. Yang, Z. N. Chen, P. Y. Lau, X. Qing, and X. Yin, "Miniaturized Patch Antenna With Grounded Strips", IEEE Transactions on Antennas and Propagation, vol. 63, no 2, pp. 843 - 848, Feb. 2015

[3] T. C. Baum, R. W. Ziolkowski, K. Ghorbani, and K. J. Nicholson, "Investigations of a Load-Bearing Composite Electrically Small Egyptian Axe Dipole Antenna", IEEE Transactions on Antennas and Propagation, vol. 65, no 8, pp. 3827 - 3837, Aug. 2017.

[4] Y.-M. Pan, K. W. Leung, and K. Lu, "Compact Quasi-Isotropic Dielectric Resonator Antenna With Small Ground Plane", IEEE Transactions on Antennas and Propagation, vol. 62, no 2, pp. 577 585, Feb. 2014

[5] S. V. Shynu, and M. J. Ammann, "Reconfigurable antenna with elevation and azimuth beam switching,", IEEE Antennas and Wireless Propagation Letters, vol. 9, pp. 367-370, 2010.

[6] M. Rutschlin, and V. Sokol, "Reconfigurable antenna simulation: Design of reconfigurable antennas with electromagnetic simulation," IEEE Microwave Magazine, vol. 14, no. 7, pp. 92-101, Nov.-Dec. 2013.

[7] A. Narbudowicz, M. J. Ammann, and D. Heberling, "Switchless Reconfigurable Antenna With $360^{\circ}$ Steering," IEEE Antennas and Wireless Propagation Letters, vol. 15, pp. 1689-1692, 2016.

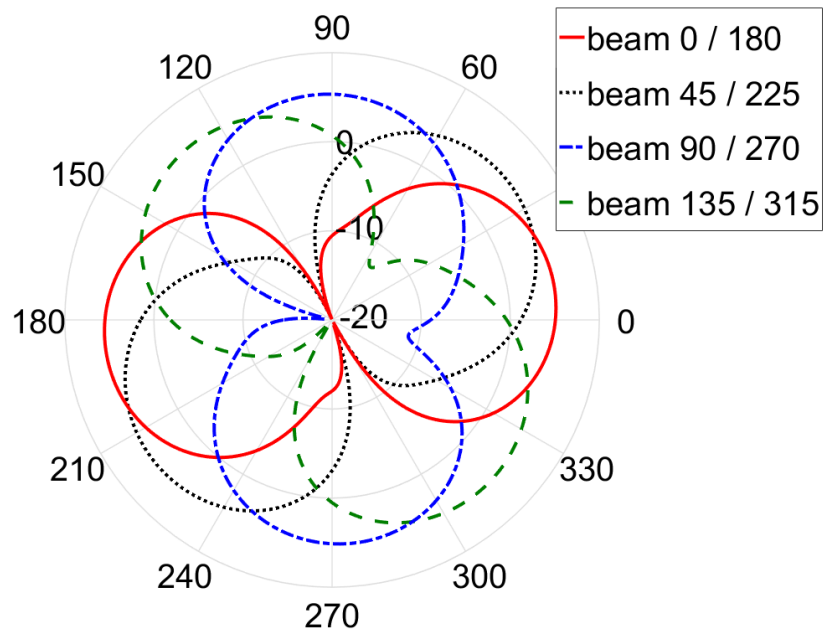

Fig. 6. Four beams synthesized with the proposed antenna by applying phase shift between its three ports.

[8] A. Narbudowicz, M. J. Ammann, and D. Heberling, "Electrically Small Antenna with Switchless Pattern Reconfiguration," In Proc.: Antennas and Propagation (APSURSI), 2017 IEEE International Symposium on, San Diego, CA, USA, July 2017.

[9] J. E. Hansen, Spherical Near-Field Antenna Measurements, P. Peregrinus, 1988.

[10] A. Narbudowicz, M. J. Ammann, and D. Heberling, "Systematic approach to inhomogeneous linear arrays," In Proc.: Antennas and Propagation (APSURSI), 2016 IEEE International Symposium on, Fajardo, PR, USA, July 2016. 